\title{
THE BULK RADIO EXPANSION OF CASSIOPEIA A
}

\author{
D.A.Green, \\ Mullard Radio Astronomy Observatory, Cavendish Laboratory, \\ Madingley Road, Cambridge CB3 OHE, United Kingdom.
}

\begin{abstract}
Comparison, in the visibility plane, of radio observations of Cassiopeia A made at $151 \mathrm{MHz}$ over a $2.3 \mathrm{yr}$ interval indicate that the bulk of the radio emitting material has not been decelerated strongly.
\end{abstract}

Introduction: Cassiopeia A (hereafter simply Cas A) is thought to be the remnant of a relatively recent $\mathrm{SN}$. Optical observations reveal, among other things, a population of fast-moving knots which show a low expansion timescale (i.e. current angular size/current angular expansion rate) of -300 yr (Kamper \& van den Bergh 1976). Interpreting these knots as undecelerated ejecta from the SN explosion (e.g. Kamper \& van den Bergh) gives an explosion date around AD1671 — indeed it has been suggested that Flamsteed may have catalogued Cas A's parent SN in AD1670 (Ashworth 1980; Kamper 1980). On the other hand, observations of compact radio knots indicate a high expansion timescale of $-950 \mathrm{yr}$ (Bell 1977; Tuffs 1986), implying material that has been decelerated appreciably. This is, at first sight, difficult to reconcile with the complex structure of the radio emission from Cas $A$ (Bell, Gull \& Kenderdine 1975), which is quite different from that expected (Gull 1975) from the blast-wave which would result if it had indeed decelerated because of sweeping up a large amount of circumstellar/interstellar material. (cf. Tycho's SN, which has decelerated considerably to near Sedov expansion (Strom, Goss \& Shaver 1982; Tan \& Gull 1985), and has a sharply-defined shell of radio emission (e.g. Duin \& Strom 1975; Green \& Gull 1983).) However, the radio knots represent only a small proportion of the total radio emission, so that the expansion timescale deduced from them is not representative of the current dynamics of the bulk of the radio emitting material.

Here I present preliminary results from a comparison of observations made of Cas $\mathrm{A}$ at $151 \mathrm{MHz}$ over an interval of $2.3 \mathrm{yr}$. These indicate an expansion timescale for the bulk of the radio emission considerably smaller than that deduced from the compact knots.

Observations: The Cambridge Low-Frequency Synthesis Telescope (CLFST) is an east-west synthesis telescope which, when operating at $151 \mathrm{MHz}$, consists of 60 multi-yagi aerials. The 28 eastern aerials (which are divided into four huts: A, B, C and D) are correlated against the 32 western aerials (huts $E, F, G$, and $H$ ) to give an almost uniform coverage of the visibility plane with baselines from $6 \lambda$ to $2352 \lambda$ in steps of $3 \lambda$. (One each of the small and large baselines are missing, and eight baselines are duplicated. Also, the aerials in hut $\mathrm{H}$ lie some way from the ideal east-west line, so that coverage of the visibility plane in regions involving baselines from these aerials is not as uniform as elsewhere.) 
Cas A was observed with the CLFST at $151 \mathrm{MHz}$ for $-12 \mathrm{hr}$ on 1984 July 18 and 1986 November 1. Expansion timescales of 300-900 yr would result in expansions of 0.77-0.26 percent between these observations, and since Cas $A$ is $-5 \operatorname{arcmin}$ in diameter, this corresponds to an expansion of the remnant of $2.3-0.8$ arcsec. Such a change is only a small fraction of the beam of the CLFST at $151 \mathrm{MHz}(-70$ arcsec), so that comparison in the map plane would require very high dynamic range maps. In practice the quality of the synthesized maps is limited by residual hut- and aerial-based calibration errors. These produce circular errors in the map plane that would contaminate, in a difficult to appreciate way, any misfit statistic used to determine the expansion of Cas A. Instead, the data have been compared in the visibility plane, where residual calibration errors can be better appreciated and the contaminated data avoided.

If there are no changes in the shape of the radio emission from Cas A between the two observations, but only an overall expansion, then the contraction of any feature in the visibility plane corresponds to the expansion of the whole of the emission from Cas A. There are, however, reports of relatively large changes ('flares') in the total radio flux of Cas A at $38 \mathrm{MHz}$ (Read 1977a, 1977b; Walczowski \& Smith 1985), although the evidence for them is marginal. These flares, which apparently occur on the timescale of a few years, must have steep spectra as they were not evident at frequencies above $38 \mathrm{MHz}$, and might be due to the 'switching-on' of the radio emission from compact features in the remnant. The comparison at $151 \mathrm{MHz}$ will be complicated if such a flare occurs during the observations.

Day-to-day variations in the gains of the aerials of the CLFST, plus the effect of varying ionospheric refraction, are usually eliminated by phase-rotating the observed visibilities to a relatively bright compact source in the field. In the case of the observations of Cas A this is not possible, but, by comparing adjacent baselines, the effects of changes in amplitude sensitivity and instrumental phase of aerials and huts relative to each other can be largely eliminated. (The relative scaling between huts $A, B, C$ and $D$ remains the most uncertain, as there are few baselines which correspond to overlap in the aperture plane between these huts.) However, due to ionospheric refraction, and poor initial phase calibration of the 1984 observations, the absolute phases, particularly for the larger baselines, are still uncertain. For these reasons, comparison of the observations has so far been restricted to the amplitude of the visibility function.

\section{Results, Conclusion (and Future Prospects)}

Results: Figure 1 showsthe amplitude of the visibility function for Cas A from the 1984 observations (several minutes' data were lost near HAs $-6^{\mathrm{h}}$ and $5^{\mathrm{h}}$ due to interference). The visibility function of an ideal shell source is centrally peaked, with circles of zero amplitude. Cas A is basically a distorted shell, and its visibility function shows a central peak, with approximately elliptical minima, the first of which is much more sharply defined than the second. The first minimum falls to less than 1 percent of the total flux density of Cas A between $\mathrm{HA} \sim 1.5^{\mathrm{h}}$ and $\mathrm{HA} \sim 3.5^{\mathrm{h}}$. Between the two observations, this well-defined portion of the first minimum shows a clear contraction corresponding to an expansion timescale of $\sim 400$ yr. This result is, however, only from regions with very low amplitude, and from a limited range of HAs, so it may be biased by the effects of flares or any shape changes. 
In order to extend the comparison to other HAs, and to regions that do not show very deep minima, a series of visibility planes were made from the 1986 data, on grids $0.1,0.2 \ldots$ per cent smaller than that used for the 1984 data. These were then compared, and a simple statistic (the relative misfit of the 1986 and 1984 amplitudes: the sum of $\left|1-\left(\operatorname{amp}_{1986} / \mathrm{amp}_{1984}\right)\right|$ over valid pixels within the region) was computed for various regions to determine the scaling change that best represents the contraction of the visibility function of Cas $A$ between the two observations.

Fortunately the baselines near first minimum all involve aerials in hut $C$, which avoids the difficulties due to calibration uncertainties between huts $A, B, C$ and $D$. The minimum misfit for a region containing the whole of the first minimum corresponds to a scale change between 0.5 and 0.6 per cent (an expansion timescale between 460 and $380 \mathrm{yr}$ ), similar to that deduced from only the very deep part of the first minimum. This result does not, however, just depend on the lowest amplitude regions or a limited HA range.

Figure 1. Visibility plane of Cas $A$ at $151 \mathrm{MHz}$ from the 1984 observations. The central peak is $-8500 \mathrm{Jy}$, and the greyscale used is (white to black in ten steps) from 0 to $2500 \mathrm{Jy}$. 
The second minimum is poorly defined, and in places spans the boundaries between huts $A$ and $B$, where relative amplitude scaling errors are still a problem. Restricting the comparison to the best defined portions of the second minimum gives a range of best-fit scale changes ranging from 0.2 percent to 0.7 percent depending on hour angle (i.e. 1150 to $330 \mathrm{yr}$ for the expansion timescale). Interpretation of these results is confused not only by residual calibration problems, but also be the range of values deduced. It is notable, however, that only one of the deeper features of the second minimum requires an expansion timescale larger than that deduced from compact radio knots.

Conclusion: These results show that, although there are variations depending on $\mathrm{HA}$, the expansion of the bulk radio emission from Cassiopeia $A$ is on a timescale nearer that deduced from the fast optical knots than that deduced from the compact radio knots, implying little deceleration of the bulk of the radio emitting material. One consequence of this low expansion timescale is that continuing particle acceleration within the remnant may not be needed (Green 1987) to explain the observed rate of decrease of its radio emission.

Future Prospects: It is hoped that the calibration of these observations (and future observations) can be be improved to a level where the effect of overall expansion of the remnant can be quantified more accurately by separating it from the HA-dependant effects due to any compact features or shape-changes. Also, with improved calibration, subtraction of synthesized maps may provide useful constraints on, or positions for any radio flares (at $151 \mathrm{MHz}$ )occurring between the observations.

Acknowledgments: I am very grateful to Dr J.A. Baldwin, with whom this work has been done in collaboration, and Mr P.J. Warner, for their help with the reduction and calibration of these observations. I am also grateful to Churchill College, Cambridge for a Junior Research Fellowship.

\section{References:}

Ashworth, W.B., 1980. J. Hist. Astr., 11, 1.

Bell, A.R. , 1977. Mon. Not. R. astr. Soc., 210, 642.

Bell, A.R., Gull \& Kenderdine, S.K., 1975. Nature, 257, 463.

Duin, R.M. \& Strom, R.G., R.G., 1975. Astr. Astrophys., 39, 33.

Green, D.A., 1987. In Genesis and Propagation of Cosmic Rays, eds Shapiro, M.M. \& Wefel, J. Reidel, Dordrecht, in press.

Green, D.A. \& Gull, S.F., 1983. In: Supernova Remnants and their X-ray emission, eds Danziger, I.J. \& Gorenstein, P., Reidel, Dordrecht, p329.

Gull, S.F., 1975. Mon. Not. R. astr. Soc., 171, 263.

Kamper, K.W., 1980. Observatory, 100, 3.

Kamper, K.W. \& van den Bergh, S., 1976. Astrophys. J. Suppl., 32, 351.

Read, P.L., 1977a. Mon. Not. R. astr. Soc., 178, 259.

Read, P.L., 1977b. Mon. Not. R. astr. Soc., 181, 63P.

Strom, R.G., Goss, W.M. \& Shaver, P.A., 1982. Mon. Not. R. astr. Soc., 200, 473.

Tan, S.M. \& Gull, S.F., 1985. Mon. Not. R. astr. Soc., 216, 579.

Tuffs, R.J., 1986. Mon. Not. R. astr. Soc., 219, 13.

Walczowski, L.T. \& Smith, K.L., 1985. Mon. Not. R. astr. Soc., 212, 27P. 